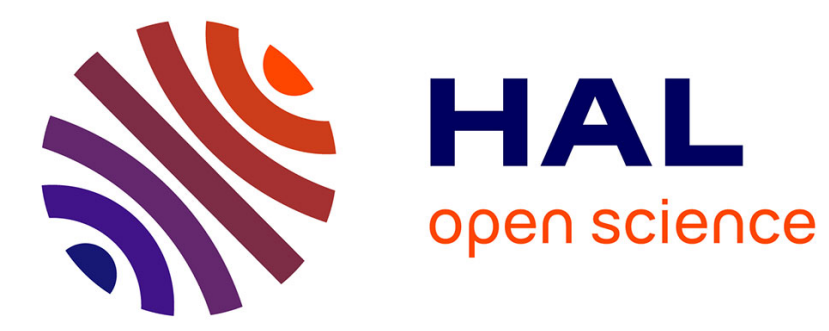

\title{
Multicriteria sorting methods to select virtual peach ideotypes
}

Mohamed-Mahmoud Memmah, Bénédicte Quilot-Turion, Antoine Rolland

\section{To cite this version:}

Mohamed-Mahmoud Memmah, Bénédicte Quilot-Turion, Antoine Rolland. Multicriteria sorting methods to select virtual peach ideotypes. International Journal of Multicriteria Decision Making, 2014, Operational Management and Marketing, 4 (4), pp.348 - 366. 10.1504/IJMCDM.2014.066874 . hal-01201746

\section{HAL Id: hal-01201746 \\ https://hal.science/hal-01201746}

Submitted on 27 May 2020

HAL is a multi-disciplinary open access archive for the deposit and dissemination of scientific research documents, whether they are published or not. The documents may come from teaching and research institutions in France or abroad, or from public or private research centers.
L'archive ouverte pluridisciplinaire HAL, est destinée au dépôt et à la diffusion de documents scientifiques de niveau recherche, publiés ou non, émanant des établissements d'enseignement et de recherche français ou étrangers, des laboratoires publics ou privés. 


\title{
Multi-criteria sorting methods to select virtual peach ideotypes
}

\section{Mohamed-Mahmoud Memmah*}

\author{
INRA, UR1115 PSH, \\ Domaine Saint Paul, \\ F-84914 Avignon Cedex 9, France \\ Email: mmouldsidi@paca.inra.fr \\ *Corresponding author
}

\section{Bénédicte Quilot-Turion}

INRA, UR1052 GAFL,

Domaine Saint Maurice,

F-84143 Montfavet Cedex 9, France

Email: quilot@avignon.inra.fr

\section{Antoine Rolland}

Laboratoire ERIC,

Université de Lyon,

69676 Bron, France

Email: antoine.rolland@lyon2.fr

\begin{abstract}
The model-based design of virtual fruit ideotypes using multi-objective optimisation algorithms could produce a high number of contrasted fruits. The breeder (decision-maker) will need an automatic tool allowing him/her to sort these contrasted ideotypes into predefined categories corresponding to several targeted traits. This paper aims to develop such a decision-making module to sort a set of fruit ideotypes into one of five preference-ordered categories in the context of brown rot-peach fruit pathosystem. First, a set of ideotypes with contrasted trade-off between three criteria was produced using multi-objective optimisation algorithms. Then, two multi-criteria decision-making methods (ELECTRE-Tri and DRSA: dominance-based rough set approach) were tested in order to reproduce the classification made by the decision-maker. Such a non-typical classification seemed difficult to be reproduced by the ELECTRE-TRI method while the decision rule-based method gave very good results (only 10\% wrong assignments). The proposed decision-making tool is very useful to speed-up the model-based design of fruit ideotypes, i.e., breeding.
\end{abstract}

Keywords: multi-criteria decision-making methods; ELECTRE-TRI; MR-sort; dominance-based rough set approach; DRSA; model-based design; ideotypes; sustainable agriculture; multi-objecitve optimisation

Reference to this paper should be made as follows: Memmah, M-M., Quilot-Turion, B. and Rolland, A. (2014) 'Multi-criteria sorting methods to select virtual peach ideotypes', Int. J. Multicriteria Decision Making, Vol. 4, No. 4, pp.348-366. 
Biographical notes: Mohamed-Mahmoud Memmah is currently a Scientist Researcher in the team 'integrated horticultural crop production' of the unit plants and cropping systems in Horticulture (UR PSH, INRA, Avignon, France). His research focuses on the model-based design of integrated horticultural production systems using nature-inspired multi-objective optimisation algorithms and multi-criteria decision-making methods.

Bénédicte Quilot-Turion is a researcher at INRA, French National Institute for Agricultural Research. She is the leader of a team working on genetic variation of peach fruit quality, using contrasted cultivars and segregating populations that are well characterised. She developed a multi-disciplinary challenging approach involving ecophysiology, genetics and computer-based modelling. Recently, she enlarged her researches towards ideotype design. Focusing on peach brown rot, she developed a model-based strategy to find the best combinations of genetic resources and cultural practices leading to conceive sustainable production systems.

Antoine Rolland received his $\mathrm{PhD}$ in Computer Science at the University Paris VI. He is Assistant Professor and Head of the Department of Statistics, in the Technology Institute, University of Lyon. His research interests are related to ordinal models in multi-criteria decision-making and links between machine learning and MCDM models. He has published research papers at national and international journals and conference proceedings.

This paper is a revised and expanded version of a paper entitled 'Méthodes multicritères pour le tri de fruits virtuels' presented at 13 eme congrès annuel de la Société française de Recherche Opérationnelle et d'Aide à la Décision, Angers, France, 11-13 April 2012.

\section{Introduction}

The complexity of agriculture production systems has lead researchers towards the use of modelling (Hammer et al., 2001; Mayer, 2002). Their challenge is to propose innovative perspectives of evolution towards systems respectful of the environment and producing safe food while ensuring the economic viability of farms. To meet this demand for multi-objective attributes, the critical question for the breeding programmes in the future is how to design best combinations of genetic resources and cultural practices adapted to, and respectful of specific environments. In other terms, how to take advantage from the strong Genotype $\times$ Environment $\times$ Management $(\mathrm{G} \times \mathrm{E} \times \mathrm{M})$ interactions in order to design plant ideotypes that meet many conflicting objectives? Following Donald (1968), we consider here an ideotype as a "plant model which is expected to perform or behave in a predictable manner within a defined environment". This virtual plant has an ideal phenotype (i.e., morphological and physiological features) that would suit a particular cropping system (Looomis, 1979). The concept of ideotype is particularly adapted:

1 in case of conflicting objectives

2 to enhance plant phenotype in particular environments. 
Designing ideotypes require knowledge in different disciplines (e.g., genetics, ecophysiology, agronomy, and pathology) which may be formalised thanks to modelling tools. Indeed, one efficient approach relies on the potentialities offered by the integration of genetic information into process-based models. The combination of genetic parameters, fingerprint of the genotype, and cultural practices are optimised to design new genotypes coupled with an adequate management, adapted to target environments (Letort et al., 2008; Tardieu, 2003).

The design of ideotypes is usually based on antagonistic criteria and subject to strong constraints (biological, economical, ecological, or environmental). The resulting fitness landscapes to be explored are often very complex. Moreover, the high number of combinations to analyse in order to identify best-adapted genotypes highlights the impossibility to exhaustively explore the whole $\mathrm{G} \times \mathrm{E} \times \mathrm{M}$ space (Messina et al., 2009). Therefore, the model-based design of ideotypes is a very difficult nonlinear multi-objective optimisation problem that resist to the classical simulation and optimisation methods.

To face this difficult multi-objective optimisation problem, an approach has recently emerged consisting in coupling process-based models (at the plant or crop level) with optimisation algorithms. Thus, bio-inspired optimisation algorithms (e.g., genetic algorithms, and particle swarm optimisation algorithms) are increasingly used for the model-based design of ideotypes (deVoil et al., 2006; Letort et al., 2008; Qi et al., 2010; Ould-Sidi and Lescourret, 2011; Kadrani et al., 2012; Quilot-Turion et al., 2012; Grechi et al., 2012). Such multi-objective evolutionary algorithms (MOEAs) allow exploring highly dimensional solution spaces in a reasonable computation time. Moreover, the MOEAs do not require any derivative information and can address the complex multi-objective optimisation problems.

Unfortunately, MOEAs focused so far on the generation of the true Pareto front and disregarded the decision-making step (Coello et al., 2007; Figueira et al., 2008). The model-based design of virtual fruit ideotypes using multi-objective optimisation algorithms could produce a high number of contrasted fruits. The breeder (decision-maker) will need an automatic tool allowing him/her to sort these contrasted ideotypes into predefined categories corresponding to several targeted traits. Coupling the MOEAs and the multi-criteria decision-making (MCDM) methods could be useful to overcome such a drawback. Indeed, sorting MCDM methods consist in helping a decision-maker to sort the different solutions into predefined categories. Research conducted in the MCDM domain has given us access to practical methods for applying scientific decision theoretical approaches to multi-criteria problems (Linkov et al., 2005). They include multi-attribute utility theory (MAUT) (Keeney and Raiffa 1976), the outranking procedure represented by the successive ELECTRE versions (Figueira et al., 2005) and PROMETHEE methods (Brans and Mareschal, 2005), and the mixed methods represented by the rule-based methods (Pawlak, 1982, 1991) and ORESTE (Roubens, 1982). The purpose of all these methods is to allow decision-makers to evaluate and choose among alternatives based on trade-offs between criteria and according to their preferences (Linkov et al., 2005; Sadok et al., 2008, 2009). Sadok et al. (2008) have reviewed the MCDM and their use in the assessment of the sustainability of alternative cropping systems. In the conclusion of their interesting paper, Sadok et al. (2008) suggest to use different MCDM methods simultaneously and to prefer the decision rule-based and outranking methods for the evaluation of the alternative cropping systems. 
In light of the above elements, this paper aims to build a decision-making model allowing to assign the ideotypes to categories in the context of peach brown rot pathosystem. Brown rot of peach fruits caused by Monilinia spp, can engender as much as $30 \%$ to $40 \%$ of crop losses. Currently, most of the cultivated peaches are more or less sensitive to brown rot. No other alternative to chemical treatment is available, hence fungicide applications are required till pre-harvest. Therefore, dealing with this storage disease is a priority to reduce fruit residues and increase food safety. Resistance to brown rot is thought to be based on complex mechanisms that are largely linked to fruit characteristics providing physical and biochemical barriers against the fungus. The apparition of cuticular cracks on the fruit surface dramatically reduces the efficiency of these barriers permitting free entrance to the pathogen in the fruit. In this context, designing peach genotypes with a low sensitivity to fruit cuticular cracks, but still of optimal organoleptic quality, is a new challenge for peach breeders. Since these objectives might be negatively correlated, different trade-offs between these objectives can be achieved and proposed by the optimisation step. Thus, the tool proposed here, interfaced with the optimisation step, will help the breeders to select the most suitable ideotypes depending on their particular objectives. For this purpose, we compare two MCDM methods:

1 a simplified version of ELECTRE-Tri (Bouyssou and Marchant, 2007a, 2007b)

which is an outranking method

2 the dominance-based rough set approach (DRSA) (Greco et al., 2001b, 2002) which is a Decision rule-based method.

A set of the ideotypes, resulting from the optimisation step, was classified by the decision-maker (a peach breeder expert) into five ordered categories based on their performances. We then proceed to the elicitation of the parameters of both methods using this learning set of ideotypes and validate the results through a bootstrap-like method. The added value of this paper is to bridge the gap between the multi-objective optimisation and MCDM communities especially in the field of model-based design of ideotypes. The tool proposed here is indeed a complementary piece in this field and will allow breeders having a real helpful modelling framework from the problem formulation till the decision-making step. The remainder of this paper is organised as follows. In the next section, we formally present the general framework of the MCDM with special emphasis on the above cited methods. Section 3 is devoted to the experimental design. In the subsequent section, we present and discuss the obtained results and we justify the choice of the retained method. Finally, we draw conclusions on this work.

\section{Multi-criteria sorting methods}

\subsection{Multi-criteria sorting methods panorama}

Three major types of MCDM problems could be treated using MCDM methods: choice, ranking, and sorting (Roy, 1996). Multi-criteria sorting problems deal with objects, named alternatives, which are described by several attributes. The aim is to assign each alternative to one or more of the predefined ordered or not ordered categories (Roy, 1996). Formally, we consider a multiple criteria sorting problem in which alternatives 
from $A=\left\{a_{1}, a_{2}, \ldots, a_{j}, \ldots, a_{m}\right\}$ are evaluated on n criteria $g_{1}, g_{2}, \ldots, g_{i}, \ldots, g_{n}$, where $i \in N=\{1, \ldots, i, \ldots, n\}$. The evaluation scale on criterion $g_{i}$ is $X_{i}$, i.e., $g_{i}: A \mapsto X_{i} . X$ denotes the Cartesian product of evaluation scales $\left(X=\prod_{i \in N} X_{i}\right)$. Predefined categories are noted $C_{1}, C_{2}, \ldots, C_{p}$ with $C_{i}$ preferred to $C_{j}$ if $i>j$. We define $C_{t}^{\geq}, t=1, \ldots, p$, $C_{t}^{\geq}=\cup_{s \geq t} C_{s}$.

Three main families of MCDM can be distinguished:

1 MAUT methods

2 the outranking methods

3 mixed or non-classical methods.

The purpose of all these methods is to allow decision-makers to evaluate and choose among alternatives based on trade-offs between criteria and according to their preferences (Linkov et al., 2005; Sadok et al., 2008, 2009). In MAUT, three main phases can be distinguished: elicitation of the decision-maker's preferences, aggregation using the appropriate global multi-attribute utility function to evaluate the expected utility of each alternative, and choice of the alternative(s) maximising the utility function (De Montis et al., 2004; Sadok et al., 2008). The preferences of the decision-maker have to be elicited in terms of:

1 set of probability distributions for outcomes associated with each alternative in each attribute

2 utility function for the range of outcomes on each attribute.

Utility functions are using scoring functions to sort the different alternatives and, in their most known form, they are based on the use of a set of weights corresponding to each criterion [decomposable utility function, used for example, in the UTADIS method (Jacquet-Lagrèze, 1995)] or to each set of criteria (for example, a Choquet integral, see Marichal, 2000). Additive function composed of generic monotonic marginal functions could also be used (Greco et al., 2008). Note that the utility functions are usually used in uncertain context while if there is no uncertainty the value functions term is used. In this last case, the MAUT is called MAVT staying for multi-attribute value theory. Thus, MAUT methods could take into account the risk on outcomes while MAVT could not. The outranking approaches for sorting problems are based on the use of the concordance and discordance principles as in ELECTRE methods (Roy, 1991; Figueira et al., 2005). Procedures based on outranking have two phases. First, the method uses the extended model of decision-makers local preferences for individual criteria including indifference, weak preference, strong preference, and incompatibility. Second, partial binary relationships such as 'alternative A is at least as good as alternative B' are established for all criteria. For example, the ELECTRE method is based on the outranking relation and comprises three steps: construction of the evaluation matrix (alternatives and criteria), calculation of the outranking relation, and exploitation of the outranking relation. The final ranking has a graphical form and is based on the outranking matrix that includes indifference, preference, anti-preference, and incompatibility (Zak, 2009). The mixed methods have no common definition within the MCDM community. This MCDM family includes methods "able to deal with mixed quantitative-qualitative or qualitative criteria 
information explicitly and/or with a preference model different from those of MAUT and outranking" following Sadok et al. (2008).

The decision to use one MCDM method rather than another should be taken by considering several aspects:

- Suitability to the studied problem: many MCDM methods are dedicated to the ranking or choosing problems. Even if it is always possible to use a ranking method to sort alternatives by the addition of thresholds for example, it is clear that MAUT methods are more efficient to rank alternatives than to sort them. Conversely, specific methods like ELECTRE Tri have been developed to sort alternatives and then should be preferably used.

- Facility of use: the use of the method should be easy even for a non-specialist; it is the case for all the methods based on decision rules.

- Proximity with an expert reasoning: after discussion with the expert, we realised that she naturally uses a kind of virtual profiles to classify the different ideotypes. This leads us to propose to use ELECTRE-Tri in order to capture these profiles and reuse them in an automatic classification. We decided also to try a decision-rule-based methods as it is also a common way for experts to decide.

Therefore, we decided to compare two different methods for sorting ideotypes: ELECTRE-Tri (or more exactly a simplified version of ELECTRE-Tri called MR-sort) and a dominance-based decision rules method using the DRSA. These methods are presented in the following subsections.

\subsection{ELECTRE-Tri}

ELECTRE TRI method (Roy, 1991, 1996; Figueira et al., 2005; Almeida-Dias et al., 2010; Doumpos et al., 2009) is a sorting method which assigns each alternative to an ordered predefined category, using ordinal comparisons with specified profiles as boundaries of the categories. We need $p-1$ profiles $q^{2}, \ldots, q^{p}$ described on $X$, as each profile is both the upper profile of a category and the lower profile of another category. We suppose that $q^{2} \prec \ldots \prec q^{p}$, and then that category 1 is the worst one and category $p$ is the best one. The first step consists in comparing an alternative to all the profiles via computing a concordance index related to each profile, i.e., the sum of the weights $\omega_{1}, \ldots, \omega_{n}$ of each criterion where the value of the alternative is considered at least as good as the value of the profile. Note that in this work we have considered a simplified version of ELECTRE-Tri which does not take into account any preference, indifference or veto threshold. This simplified version of ELECTRE-Tri is known as the majority rule sorting procedure (MR-sort) and has been characterised by Bouyssou and Marchant (2007a, 2007b). We now have for each alternative $a \in A$ a concordance index related to each of the profiles: $C I\left(a, q^{i}\right)=\sum_{j \mid g_{j}(a) \geq g_{j}\left(q^{i}\right)} \omega_{j}$. We then use a cutting level $\lambda$ to obtain a crisp preference relation on each pair alternative/profile: $a \succsim q^{i} \Leftrightarrow C I\left(a, q^{i}\right) \geq \lambda$. The last step consists in assigning a category to each alternative based on the preference relation to the profiles. ELECTRE-Tri considers two different ways of assignment: the pessimistic assignment of ELECTRE TRI consists in comparing the alternative to be classified with profiles raising gradually; the first profile whom the alternative 
is not preferred to gives the category where to classify the alternative, i.e., $q \in C_{i} \Leftrightarrow\left[\left(a \succsim g^{i}\right)\right.$ and $\left.\left(a \succsim q^{i+1}\right)\right]$. On the opposite, the optimistic assignment of ELECTRE TRI consists in comparing the alternative to be classified with profiles going down: the first profile which is not preferred to the alternative gives the category where to classify the alternative, i.e., $a \in C_{i} \Leftrightarrow\left[\left(q^{i+1} \succ a\right)\right.$ and $\left.\left(q^{i} \nsucc a\right)\right]$. Note that in MS-Sort both optimistic and pessimistic assignments lead to the same assignment.

\subsection{Decision rules induced through DRSA}

This method consists in using decision rules through the DRSA. The DRSA (Greco et al., $2001 \mathrm{~b}, 2002$ ) is a generalisation of the classical rough set approach (CRSA) (Pawlak, $1982,1991)$ to be usable in the framework of the MCDM. Unlike the CRSA, DRSA is able to deal with inconsistency concerning violation of the dominance principle typical exemplary decisions in MCDM problems (Greco et al., 2005). Alternatives are assigned to the different categories with respect to some reference levels on each criterion. Greco et al. (2001a) have well studied the axiomatic foundations of the rough set approach with special emphasis on the characterisation of the sorting problem using a utility function, an outranking relation or a Sugeno integral (Słowiński et al., 2002). Formally, a decision rules model is able to sort alternative $a$ thanks to rules like 'if $g_{1}(a)>\alpha_{1}$ and $g_{2}(a)<\alpha_{2}$ and ... then a is sorted in category $C_{i}$ '. The learning procedure of the decision rules is based on DRSA on a learning data set, as described in Greco et al. (2000). Given the set of criteria $\left\{g_{i}, i \in N\right\}$, the inclusion of an alternative $a \in A$ to the upward union of classes $C_{t}^{\geq}, t=2, \ldots, p$ creates an inconsistency in the sense of dominance principle if one of the following conditions holds:

- $\quad a$ belongs to class $C_{t}^{\geq}$or better but it is dominated by an alternative $\mathrm{b}$ belonging to a class worse than $C_{t}$

- $a$ belongs to a worse class than $C_{t}^{\geq}$but it dominates an alternative $\mathrm{b}$ belonging to class $C_{t}$ or better

If the inclusion of $a \in A$ to $C_{t}^{\geq}, t=2, \ldots, p$, creates an inconsistency in the sense of dominance principle, then $a$ belongs to $C_{t}^{\geq}$with some ambiguity. Thus, $a$ belongs to $C_{t}^{\geq}$ without any ambiguity if $a \in C_{t}^{\geq}$and there is no inconsistency in the sense of dominance principle. This means that all alternatives dominating $a$ belong to $C_{t}^{\geq}$.

The assignment of the alternatives belonging to the learning data sets can then be distinguished between certain assignments and possible assignments. These assignments then induce certain decision rules and possible decision rules. Assignment of new alternatives is then made using the certain decision rules.

\section{Experimental design}

\subsection{Case study: criteria, alternatives, and decision-making}

As previously mentioned, we used a model-based approach to design peach ideotypes with enhanced values for fruit quality and resistance aspects (brown rot sensitivity) and 
adapted to given cultural scenarios. This approach coupled the 'virtual fruit' (Lescourret and Génard, 2005; Génard et al., 2007, 2010), a process-based model which simulates peach growth, and the well-known MOEA NSGA-II (Deb et al., 2002). We focused the work on six parameters of the model identified via a sensitivity analysis, to be combined to create the genotypes (a set of the six parameters). Three traits (criteria) simulated by the model and of major importance for fruit quality and sensitivity to brown rot were taken into account to evaluate the genotypes. Simulations were performed in weather conditions of Avignon (France) in 2009, in case of well-irrigated conditions and low crop load (number of fruits) of the trees.

\subsubsection{Criteria}

A large number of selection goals and criteria are taken into account during the breeding process, such as tree vigour and easy training, floribondity, harvest calendar and yield, fruit mass and fruit quality, disease resistance. Besides traits of agronomic interest, fruit appearance and sweetness are by far the main determinants in the choice of the consumers. In addition, health aspects (lower pesticide residues) are growing concerns. However, currently fruit mass is still one of the most important fruit criteria considered by retailers and therefore by producers, for the sake of profitability. Alternative breeding schemes should be considered for future, favouring organoleptic quality or environment friendly practices. However, breeders have first to cope with a major difficulty that resides in adverse correlations between fruit characteristics, in particular between fruit mass, fruit cracking, and sweetness. In this context, our study focused on fruit mass, sweetness and skin density of cracks (tightly linked to sensitivity to brown rot). The fruit mass and sweetness have to be maximised while the density of cracks has to be minimised since it is considered as an open door for the brown rot pathogen.

\subsubsection{Alternatives}

The approach coupling the 'virtual fruit' model and the NSGA-II algorithm produces a large diversity of solutions or alternatives. Indeed, the optimisation algorithm generates a population of Pareto-optimal solutions (genotypes) at each run. As this algorithm uses, like any heuristic, stochastic mechanisms the simulations need to be repeated. Thus, the number of produced solutions at the end of the process (virtual ideotypes) could be very high. Any ideotype produced by the above mentioned approach is considered as an alternative and has to be compared with the other ideotypes. Most of the alternatives were distributed along Pareto front (suggesting a good convergence of the algorithm). In this case, neighbouring alternatives are very similar. They represent a same ideotype (selection goal). In addition, some solutions were located in non-crowded zones and constitute some original alternatives for the final decision-maker. Overall, the alternatives displayed highly contrasted trade-offs between the three criteria. For example, a big fruit has more chance to have many cracks and to be sweeter than a small one. Some alternatives may be 'balanced fruits' with good attributes for all the three criteria and 'oriented fruits' excellent for a single criterion and merely good for the two others. Finally, the decision-maker can choose a type of alternatives that best suit the trade-off between criteria according to his/her particular objective. However, the choice of the alternatives may rapidly become long, repetitive and laborious, especially when the number of alternatives increases with simulations in different sites or climatic conditions. 
This largely justifies the need of an automatic tool allowing him/her to sort the alternatives into predefined categories.

\subsubsection{Decision-making}

The case study we propose here deals with 120 alternatives stemmed from the optimisation algorithm coupled to the 'virtual fruit' applied in Avignon weather conditions of a given year. The 120 alternatives have been classified into five ordered categories by a single peach breeder expert (the decision-maker). The worst category is denoted by 1 , the best one is denoted by 5 . The choice to consider only one decisionmaker in this study is for sake of simplicity. Even if breeders could have different points of view regarding ideotypes, we decided in this preliminary work to limit our investigations to the MCDM and to consider multi-actor multi-criteria decision-making (MA-MCDM) in our future work. The decision of considering five categories was taken by the expert in order to differentiate the ideotypes according to combinations of the three targeted traits (criteria). Indeed, fruits could be small and acid, having large cracks' density, or be sweet and big, or sweet and having a small cracks' density, etc. The five categories represent five contrasted putative selection goals (or ideotypes) and the alternatives within each category represent different genotypes that may reach the goal.

Table 1 Range of the criteria

\begin{tabular}{lcc}
\hline Criterion & min & max \\
\hline Fruit mass (g) & 108 & 300 \\
Sweetness (\%) & 5 & 20 \\
Density of no crack (\%) & 82 & 100 \\
\hline
\end{tabular}

Table 2 Discretisation of the values of the criteria

\begin{tabular}{ll}
\hline Criterion & Transformation \\
\hline MF & Rounded to the nearest multiple of $15(105,120, \ldots, 300)$ \\
SW & Rounded to the nearest integer $(5,6, \ldots, 20)$ \\
DC & Rounded to the nearest integer $(81,82, \ldots, 100)$ \\
\hline
\end{tabular}

As a result, the decision-maker, integrating the complexity of the system in its reasoning, proposed a classification that may appear tricky. In this classification, no criterion was determinant alone since some big or very sweet or no cracking fruits were assigned by the decision-maker in the worst category. On the contrary, fruits having only average performances (small, more or less sweet, no negligible crack density) were assigned to the best categories. This point could be explained by the conflict between criteria and the importance of the trade-offs between them. Also, a sort of veto threshold seems to emerge from the decision-maker reasoning. Indeed, no simple rules can be caught at a glimpse and sophisticated methods are necessary to reproduce this classification. The whole data set (120 ideotypes) has been pretreated in order to prepare the elicitation of the parameters of each MCDM method. Thus, the last criterion (density of crack) was transformed into density of no-crack (DC) in order to have three criteria to be maximised. Even if this transformation is not required neither by optimisation algorithm nor by used MCDM methods, it seems more interesting for the users and decision-makers. The range of variations of the three criteria within the 120 alternatives is presented in Table 1. In 
addition, the evaluation interval of each criterion was divided into different levels (Table 2) in order to facilitate the expert decision (and then the elicitation of parameters by each method) by avoiding any question about potential indifference or preference thresholds.

\subsection{Implementation of the two MCDM methods}

\subsubsection{ELECTRE Tri}

The implementation of the two MCDM methods means the determination of the values of preference parameters for ELECTRE-TRI and inferring decision-rules for the DRSA approach. As stated by Figueira et al. (2005), this preference elicitation should be an interactive process between decision-makers and analysts. Based on the information given by decision-makers, this process has to be carried out so that each method reproduces as possible the decision-maker sorting. Two types of elicitation procedures could be distinguished depending on the expression of the decision-makers preferences: direct and indirect. In the first case, decision-makers could express their preferences in the form of assertions on the values of the preference parameters. In the second type, the values of the parameters are inferred from examples (i.e., assignment examples). According to Figueira et al. (2005), ELECTRE methods are usually implemented using the indirect elicitation procedures since it is difficult to understand the precise meaning of the assertions of decision-makers in direct elicitation techniques.

Elicitation procedures of the parameters of ELECTRE-Tri method have been developed by many authors (see Figueira et al., 2005). These procedures could be used to infer all (complete inference) or subset (partial inference) of the preference parameters. In real-world problems, due to the complexity of the induced mathematical model to be solved in order to elicit the parameters, the partial inference is preferred. Thus, we can infer for examples: concordant coalition parameters (weights and cutting level); discordance related parameters (veto threshold); category limits.

The elicitation algorithm used in this work to infer the parameters of MR-sort has been proposed by Sobrie et al. $(2012,2013)$. The parameters of MR-sort to be elicited are the performance vector of profiles, the criteria weights and the majority threshold. This algorithm was developed for problems involving a large number of variables as in our case study (large number of alternatives generated by optimisation, five categories, and three criteria). Indeed, as stated by Sobrie et al. (2012), inferring the MR-sort parameters for such problems by linear programming is time consuming due to the high number of necessary binary variables for profiles evaluations. Therefore, Sobrie et al. (2012) propose a population-based metaheuristic to infer MR-sort's parameters within a reasonable computational time. Their algorithm could be decomposed into the following steps. First, it initialises the profiles using a heuristic. Then, considering the current profiles, it computes the weights and the majority threshold solving a linear programme. Third, a dedicated heuristic is used to adjust the profiles using weights and a majority threshold computed in step 2. Next, evaluation of all candidate MR-sort models is done and the worst ones (half) are reinitialised. The evaluation criterion used by the algorithm is the classification accuracy measured by the ratio between the number of true assignments and the total number of assignment examples. Steps, except initialisation, are iteratively repeated. The algorithm stops if a maximum number of iterations is attained or 
if at least one model in the population has a classification value equal to 1 . The number of profiles was determined by the number of classes.

The inputs of the partial elicitation procedure used in this work are:

- a set of alternatives and their categories, which is the learning set

- a set of criteria: MF, SW and DC

- the performance table of the alternatives on each criterion.

\title{
3.2.2 Decision rules induced through DRSA
}

The induction of decision rules is a very difficult problem usually tackled using heuristics (Greco et al., 2005). Stefanowski (1998a) distinguishes three types of rough set-based algorithms respectively inducing minimum, exhaustive, and satisfactory sets of rules. As indicated by their names, the first type of algorithms aims to generate the smallest number of rules describing the inputs while the second one tries to induce all possible decision rules. The third type could be considered as a sort of trade-off between the first and the second type since it tries to generate a set of rules satisfying the decision-maker preferences. A large number of heuristics and software systems are available for inducing decision rules from examples. LEM2, MODLEM, and DOMLEM algorithms and LERS, RoughDAS, RoughFamilly systems are examples among others of such algorithms and software systems. Interested readers could consult Stefanowski (1998a), Greco et al. (2001b, 2005) for more details. To induce the decision rules which are able to sort the fruits in the desired categories, we used the JMAF software (Greco et al., 2002; Błaszczyński et al., 2013). JMAF is a well-known, free, and easy to use software which implements the DOMLEM and DOMLEM-VC algorithms (Greco et al., 2005). DOMLEM is the first known rule induction algorithm, with polynomial complexity, developed for multi-criteria sorting problems. Therefore, this algorithm is very suitable for our case study. DOMLEM is a heuristic developed to generate a minimal (complete and non-redundant) set of decision rules. The main procedure of DOMLEM, proposed in (Greco et al., 2005), is based on the concept of MODLEM (Stefanowski 1998b), described by Stefanowski (2002):

\begin{abstract}
"It is based on the scheme of a sequential covering and it heuristically generates a minimal set of decision rules for every decision concept (decision class or its rough approximation in case of inconsistent examples). Such a set of rules attempts to cover all (or the most significant) positive examples of the given concept and not to cover any negative examples (or as little as possible of them). The main procedure for rule induction scheme starts from creating a first rule by choosing sequentially the 'best' elementary conditions according to chosen criteria.[...] When the rule is stored, all learning positive examples that match this rule are removed from consideration. The process is iteratively repeated while some significant positive examples of the decision concept remain still uncovered. Then, the procedure is sequentially repeated for each set of examples from a succeeding decision concept".
\end{abstract}

\subsubsection{Testing procedure}

We inferred the decision rules on the learning set of 120 examples. We divided the set into ten equal parts. We then get one part out of the learning set, and use the 108 examples left to elicit the parameters of the model. We then test the parametrised 
model on the 12 other examples. We check for each fruit the difference between the category given by the expert and the category obtained by the model. We repeat this procedure ten times, changing of testing set each time.

\section{Results and discussion}

As mentioned in the previous section, we used the algorithm proposed in Sobrie et al. $(2012,2013)$ to determine the parameters of ELECTRE tri: the limit profiles of the categories, the weights and the cutting level. Table 3 presents an example of inferred profiles. The weights have been inferred to follow the majority rule, i.e., all the weights are fixed at $1 / 3$ and the cutting level at $1 / 2$. The confusion matrix presented in Table 4 shows the links between the initial category assigned by the expert (rows) and the category assigned by the used procedure (columns). This matrix gives us a clear idea of the accuracy of the proposed classification. Ideally, we expect a diagonal matrix which means that each alternative was assigned to the same category by both the used algorithm and the decision-maker. The mentioned table shows that 16 alternatives have been assigned to the category 3 by the algorithm while the expert had assigned them to the category 1 . Similarly, five alternatives belonging to the category 4 according to the expert have been assigned to the category 3 by the procedure. Consequently, performances are not so good as only $62.5 \%$ of the ideotypes have been well classified by ELECTRE Tri. In most cases of wrong classification, the algorithm had upgraded the alternatives. This might be due to a 'veto' threshold the decision-maker had imposed to some alternatives: even though two criteria have excellent values, if the third one was too bad the decision-maker classified them to low levels. Indeed, it seems the decision-maker has applied implicit and redhibitory minimum levels that the algorithm failed to capture.

A further analysis of the preferences of the decision-maker showed that it seems like the inferred floating veto thresholds depend on the values of the other criteria. This cannot be modelled by the used version of ELECTRE-Tri (MR-sort) method since it does not consider any type of threshold as indicated in the Subsection 2.2. It was therefore difficult for the elicitation process to infer the parameters of MR-sort suitable for the classification of the decision-maker.

We inferred the decision rules using the DOMLEM through the JMAF software. The number of inferred decision rules is varying between 24 and 28 depending on the learning set. One example is detailed in the appendix. The obtained results show that the decision rules inferred through the DRSA using the DOMLEM elicitation procedure was able to capture the decision-maker preferences and lead to $79 \%$ of good assignments, $10 \%$ of wrong assignments and $11 \%$ of no assignments. In addition, the number of rules seems to be reasonable and the rules were easily interpretable by the decision-maker. The confusion matrix (Table 5) shows the links between the initial category and the category given by the procedure. For example in this matrix, one of the fruits classified in category 4 by the decision-maker has been sorted in category 5 by the use of decision rules, and four of them have not been classified by the decision rules. Most of the wrong classifications corresponded to 1 level difference only. However, one problematic result come from the two alternatives classified in category 5 by the decision rules whereas the decision-maker had sorted them in category 1 . These two alternatives have the particularity to display very high levels of crack density that have been redhibitory by the 
decision-maker despite their very good status for fruit mass and sweetness. Most of the non-classified alternatives belong to the middle classes: they are not very good nor very bad. In conclusion, this method proved very efficient to pinpoint the extreme alternatives with a rate of significant error of only $2 / 57$. This automatic classification may help the decision-maker to eliminate the deficient alternatives and single out the best ones. Only two alternatives over 36 meant to be of level 5 by the decision rules would not reach the expectations. Moreover, no alternative classified in level 5 by the decision rules would have deserved to be retained.

Table 3 ELECTRE-TRI profiles elicited by the use of an interactive linear programming

\begin{tabular}{lccc}
\hline Profile & $M P$ & $S W$ & $D C$ \\
\hline P1 & 150 & 5 & 82 \\
P2 & 160 & 7 & 85 \\
P3 & 190 & 11 & 93 \\
P4 & 210 & 11 & 97 \\
\hline
\end{tabular}

Table 4 Confusion matrix with ELECTRE-Tri

\begin{tabular}{cccccc}
\hline & \multicolumn{5}{c}{ Learning set } \\
\cline { 2 - 6 } & 1 & 2 & 3 & 4 & 5 \\
\hline 1 & 0 & 3 & 16 & 0 & 0 \\
2 & 0 & 0 & 3 & 9 & 1 \\
3 & 0 & 0 & 20 & 3 & 2 \\
4 & 0 & 0 & 5 & 18 & 2 \\
5 & 0 & 0 & 0 & 1 & 37 \\
\hline
\end{tabular}

Notes: In rows, categories assigned by the decision-maker. In columns, categories assigned by the algorithm.

Table 5 Confusion matrix with DRSA

\begin{tabular}{ccccccc}
\hline & \multicolumn{7}{c}{ Learning set } \\
\cline { 2 - 7 } & 1 & 2 & 3 & 4 & 5 & Non-classified \\
\hline 1 & 14 & 1 & 2 & 0 & 2 & 0 \\
2 & 0 & 10 & 0 & 0 & 0 & 2 \\
3 & 0 & 0 & 20 & 0 & 0 & 6 \\
4 & 0 & 0 & 1 & 18 & 1 & 4 \\
5 & 0 & 0 & 0 & 5 & 33 & 1 \\
\hline
\end{tabular}

Notes: In rows, categories assigned by the decision-maker. In columns, categories assigned by the algorithm.

The results presented in this work are in agreement with the statements of Leroy et al. (2011), Figueira et al. (2005) and Greco et al. (2001b).

The ELECTRE-Tri has failed to capture the decision-maker preferences in our case study. Leroy et al. (2011) pointed out the complexity of the problem of inferring the parameters of the original ELECTRE-Tri version. According to them, the high number of parameters and the nonlinear constraints are hardly handled by the proposed methods. Based on this observation, they used the same simplified version as the one used in this 
work (i.e., MR-sort method) and inferred simultaneously all its parameters. With a learning set of up to 100 alternatives involving up to five criteria and three profiles, they were able to solve the corresponding nonlinear mixed integer programme in few seconds with the CPLEX software. However, authors mentioned that the mixed integer programme formulating the parameters inferring problem happened to be with no feasible solution in case of alternatives incompatible with their MR-sort method.

The failure of the ELECTRE-TRI in our application case could also be explained by the nature of the studied problem. Indeed, as stated by Figueira et al. (2005) ELECTRE methods should be used only to deal with decision-making problems having at least three criteria but their high performance is usually obtained when decision models include more than five criteria (up to 12 or 13) due to their aggregation procedures.

In perspective of considering more criteria for the future breeding schemes, such as method could be more competitive. Even if in this study, we limited our investigations to three criteria of major interest for peach breeders in the brown rot context, a large number of criteria and goals are usually taken into account during the breeding process. A combination of different decision-making methods could be used at different steps of the breeding, depending on the number of criteria studied at each step.

Another interesting perspective that might improve the performances of ELECTRE-Tri is to consider others versions of this method taking into account veto and preference thresholds. Such an improvement might help us to infer the implicit thresholds considered by the expert in this classification but requires the use of more powerful elicitation algorithms. Indeed, the number of variables to be inferred shall increase considerably in this new situation. Greco et al. (2001b) confirmed that the DRSA may be of broader use than MAUT and the outranking methods such as ELECTRE-Tri. In the rough set approach, the decision-maker expresses his/her preferences in a natural way giving exemplary decisions without any explanation in terms of specific parameters. This approach does not require numerous parameters unlike other multi-criteria decision methods. In addition, the last but not least argument to choose this approach is the straightforward interpretation of the inferred decision rules.

The DRSA was adapted to solve the application case of selection of virtual peach ideotypes. The resulting classification was accurate in the face of the complexity of the initial classification proposed by the decision-maker. In addition, the rough set approach underlined some inconsistencies of the initial classification and the decision-maker well received the proposed modifications. Such multi-criteria sorting methods have been used at different levels in agricultural and environmental decision-making to help trade-off the economic, environmental, and social aspects (Dooley et al., 2009). However, to our knowledge this work is the first one at the level of the plant to help rationalise ideotype conception. At the end, there is high interest in extending the scope of application of these multi-criteria sorting methods towards the completion of a pipeline coupling process-based models and optimisation methods in order to speed-up the breeding programmes in the future. As mentioned in the decision-making subsection, we implicated one expert only in this study. This might be one of the limitations of this work but we did so for the sake of simplicity in this preliminary study. It is very important in the future to consider a MA-MCDM methodology to take explicitly into account a multiplicity of stakeholders' opinions. This perspective implies to develop an approach searching for consensus between the breeders [see for example, Eisa (2013) and Cailloux et al. (2012) for recent works about group decision with DRSA and ELECTRE-Tri]. 


\section{Conclusions}

We have studied two multi-criteria sorting methods in the framework of virtual peach ideotypes selection. These methods were: ELECTRE-Tri and decision rules induced using DRSA. This work has two potential uses oriented towards:

1 the decision-maker

2 the modeller.

For the decision-maker, the interest is to obtain an automatic classification of the genotypes in accordance with a set of ideotypes he had classified once. The modeller aims to classify thousands of optimal solutions resulting from simulations in different climatic and cultural practices scenarios and thus to unload and help the so busy decision-maker by inferring his/her preferences. Only the decision rules-based method proved to be efficient to represent the preferences of the decision-maker. This was mainly due to the difficulty to choose the required parameters for ELECTRE-Tri. The natural spirit of rough set approach may also be invoked. For further research, it could be interesting to involve more than one decision-maker in order to compare and confront their points of view.

\section{Acknowledgements}

Authors would like to thank the anonymous reviewers for their comments that help them to improve the quality of this paper.

\section{References}

Almeida-Dias, J., Figueira, J. and Roy, B. (2010) 'ELECTRE tri-C: a multiple criteria sorting method based on characteristic reference actions', European Journal of Operational Research, Vol. 204, No. 3, pp.565-580.

Błaszczyński, J., Greco, S., Matarazzo, B., Słowiński, R. and Szelag, M. (2013) 'JMAF dominance-based rough set data analysis framework', in Skowron, A. and Suraj, Z. (Eds.): Rough Sets and Intelligent Systems - Professor Zdzislaw Pawlak in Memoriam, pp.185-209, Springer.

Bouyssou, D. and Marchant, T. (2007a) 'An axiomatic approach to noncompensatory sorting methods in MCDM, I: the case of two categories', European Journal of Operational Research, Vol. 178, No. 1, pp.217-245.

Bouyssou, D. and Marchant, T. (2007b) 'An axiomatic approach to noncompensatory sorting methods in MCDM, II: more than two categories', European Journal of Operational Research, Vol. 178, No. 1, pp.246-276.

Brans, J. and Mareschal, B. (2005) 'PROMETHEE methods', in Figueira, J., Greco, S. and Ehrgott, M. (Eds.): Multiple Criteria Decision Analysis: State of the Art Surveys, pp.163-196, Springer Verlag.

Cailloux, O., Meyer, P. and Mousseau, V. (2012) 'Eliciting ELECTRE TRI category limits for a group of decision makers', European Journal of Operational Research, Vol. 223, No. 1, pp.133-140.

Coello, C., Veldhuizen, D. and Lamont, G. (2007) Evolutionary Algorithms for Solving Multi-Objective Problems, Springer, New York. 
De Montis, A., de Toro, P., Doste-Franke, B., Omann, I. and Stagl, S. (2004) 'Assessing the quality of different MCDA methods', in Getzner, M., Spash, C. and Stagl, S. (Eds.): Alternatives for Environmental Valuation, pp.99-133, Rouledge.

Deb, K., Pratap, A., Agarwal, S. and Meyarivan, T. (2002) 'A fast and elitist multiobjective genetic algorithm: NSGA-II', IEEE T. Evolut. Computat., Vol. 6, No. 2, pp.182-197.

deVoil, P., Rossing, W. and Hammer, G. (2006) 'Exploring profit - sustainability trade-offs in cropping systems using evolutionary algorithms', Environmental Modelling \& Software, Vol. 21, No. 9, pp.1368-1374.

Donald, C-M. (1968) 'The breeding of crop ideotypes', Euphytica, Vol. 17, No. 3, pp.385-403.

Dooley, A., Smeaton, D., Sheath, G. and Ledgard, S. (2009) 'Application of multiple criteria decision analysis in the New Zealand agricultural industry', Journal of Multi-Criteria Decision Analysis, Vol. 16, Nos. 1-2, pp.39-53.

Doumpos, M., Marinakis, Y., Marinaki, M. and Zopounidis, C. (2009) 'An evolutionary approach to construction of outranking models for multicriteria classification: the case of the electre tri method', European Journal of Operational Research, Vol. 199, No. 2, pp.496-505.

Eisa, M. (2013) 'Improving group decision support systems using rough set', International Journal of Computer Applications, Vol. 69, No. 2, pp.9-13.

Figueira, J., Greco, S., Mousseau, V. and Słowiński, R. (2008) 'Interactive multiobjective optimization using a set of additive value functions', in Branke, J., Deb, K., Miettinen, K. and Słowiński, R. (Eds.): Multiobjective Optimization, pp.97-119, Springer Verlag, Berlin/Heidelberg.

Figueira, J., Mousseau, V. and Roy, B. (2005) 'ELECTRE methods', in Figueira, J., Greco, S. and Ehrgott, M. (Eds.): Multiple Criteria Decision Analysis: State of the Art Surveys, pp.133-153, Springer Verlag, Boston, Dordrecht, London.

Génard, M., Bertin, N., Borel, C., Bussières, P., Gautier, H., Habib, R., Lechaudel, M., Lecomte, A., Lescourret, F., Lobit, P. and Quilot-Turion, B. (2007) 'Towards a 'virtual fruit' focusing on quality: modelling features and potential uses', Journal of Experimental Botany, Vol. 58, No. 5, pp.917-928.

Génard, M., Bertin, N., Gautier, H., Lescourret, F. and Quilot-Turion, B. (2010) 'Virtual profiling: a new way to analyse phenotypes', Plant Journal, Vol. 62, No. 2, pp.344-355.

Grechi, I., Ould-Sidi, M-M., Hilgert, N., Senoussi, R., Sauphanor, B. and Lescourret, F. (2012) 'Designing integrated management scenarios using simulation-based and multi-objective optimization: application to the peach tree-myzus persicae aphid system', Ecological Modelling, Vol. 246, No. 11, pp.47-59.

Greco, S., Matarazzo, B. and Słowiński, R. (2000) Multicriteria Classification by DominanceBased Rough Set Approach, Methodological Basis of the 4emka System [online] http://wwwidss.cs.put.poznan.pl/4emka/ (accessed 30 May 2014).

Greco, S., Matarazzo, B. and Słowiński, R. (2001a) 'Conjoint measurement and rough set approach for multicriteria sorting problems in presence of ordinal criteria', in Colorni, A., Paruccini, $M$. and Roy, B. (Eds.): A-MCD-A, Aide Multicritère à la Décision/Multiple Criteria Decision Aid, European Commission, Joint Research Centre, EUR 19808 EN, Ispra, pp.117-144.

Greco, S., Matarazzo, B. and Słowiński, R. (2001b) 'Rough sets theory for multicriteria decision analysis', European Journal of Operational Research, Vol. 129, No. 1, pp.1-47.

Greco, S., Matarazzo, B. and Słowiński, R. (2002) 'Rough sets methodology for sorting problems in presence of multiple attributes and criteria', European Journal of Operational Research, Vol. 138, No. 2, pp.247-259.

Greco, S., Matarazzo, B., Słowiński, R. and Stefanowski, J. (2005) 'An algorithm for induction of decision rules consistent with the dominance principle', in Ziarko, W. and Yao, Y. (Eds.): Rough Sets and Current Trends in Computing, pp.304-313, Springer, Berlin/Heidelberg.

Greco, S., Mousseau, V. and Słowiński, R. (2008) 'Ordinal regression revisited: multiple criteria ranking using a set of additive value functions', European Journal of Operational Research, Vol. 191, No. 2, pp.416-436. 
Hammer, G., Hansen, J., Phillips, J., Mjelde, J., Hill, H., Love, A. and Potgieter, A. (2001) 'Advances in application of climate prediction in agriculture', Agricultural Systems, Vol. 70, Nos. 2-3, pp.515-553.

Jacquet-Lagrèze, E. (1995) 'An application of the UTA discriminant model for the evaluation of SC R\&D projects', in Pardalos, P., Siskos, Y. and Zopounidis, C. (Eds.): Advances in Multicriteria Analysis, Nonconvex Optimization and its Applications, pp.203-211, Kluwer Academic, Dordrecht.

Kadrani, A., Ould-Sidi, M-M., Quilot-Turion, B., Génard, M. and Lescourret, F. (2012) 'Particle swarm optimization to design ideotypes for sustainable fruit production systems', International Journal of Swarm Intelligence Research, Vol. 3, No. 2, pp.1-19.

Keeney, R. and Raiffa, H. (1976) Decisions with Multiple Objectives: Preferences and Value Tradeoffs, J. Wiley, New York.

Leroy, A., Mousseau, V. and Pirlot, M. (2011) 'Learning the parameters of a multiple criteria sorting method based on a majority rule', in 2nd International Conference on Algorithmic Decision Theory, DIMACS, Rutgers University, New Jersey, 26-28 October.

Lescourret, F. and Génard, M. (2005) 'A virtual peach fruit model simulating changes in fruit quality during the final stage of fruit growth', Tree Physiology, Vol. 25, No. 10, pp.1303-1315.

Letort, V., Mahe, P., Cournede, P., De Reffye, P. and Courtois, B. (2008) 'Quantitative genetics and functional-structural plant growth models: simulation of quantitative trait loci detection for model parameters and application to potential yield optimization', Annals of Botany, Vol. 101, No. 8, pp.1243-1254.

Linkov, I., Varghese, A., Jamil, S., Seager, T., Kiker, G. and Bridges, T. (2005) 'Multi-criteria decision analysis: a framework for structuring remedial decisions at contaminated sites', Comparative Risk Assessment and Environmental Decision Making, NATO Science Series: IV: Earth and Environmental Sciences, Vol. 38, pp.15-54.

Looomis, R-S. (1979) 'Ideotype concepts for sugarbeet improvement', Journal of American Society for Sugar Beet Technologies, Vol. 20, No. 4, pp.323-342.

Marichal, J-L. (2000) 'An axiomatic approach of the discrete Choquet integral as a tool to aggregate interacting criteria', IEEE Transactions on Fuzzy Systems, Vol. 8, No. 6, pp. $800-807$.

Mayer, D. (2002) Evolutionary Algorithms and Agricultural Systems, Kluwer Academic, Dordrecht.

Messina, C., Hammer, G., Dong, Z., Podlich, D. and Cooper, M. (2009) 'Modelling crop improvement in a GxExM framework via gene-trail-phenotype relationships', in Sadras, V.O. and Calderini, D. (Eds.): Crop Physiology: Applications for Genetic Improvement and Agronomy, pp.235-265, Elsevier.

Ould-Sidi, M-M. and Lescourret, F. (2011) 'Model-based design of integrated production systems: a review', Agronomy for Sustainable Development, Vol. 31, No. 3, pp.571-588.

Pawlak, Z. (1982) 'Rough sets', International Journal of Information and Computer Sciences, Vol. 11, No. 5, pp.341-356.

Pawlak, Z. (1991) Rough Sets. Theoretical Aspects of Reasoning about Data, Kluwer, Dordrecht.

Qi, R., Ma, Y., Hu, B., de Reffye, P. and Cournede, P. (2010) 'Optimization of source-sink dynamics in plant growth for ideotype breeding: a case study on maize', Computers and Electronics in Agriculture, Vol. 71, No. 1, pp.96-105.

Quilot-Turion, B., Ould-Sidi, M-M., Kadrani, A., Hilgert, N., Génard, M. and Lescourret, F. (2012) 'Optimization of genetic parameters of the 'virtual fruit' model to design peach ideotypes for sustainable production systems', European Journal of Agronomy, Vol. 42, No. 7, pp.34-48.

Roubens, M. (1982) 'Preference relations on actions and criteria in multicriteria decision making', European Journal of Operational Research, Vol. 10, No. 1, pp.51-55.

Roy, B. (1991) 'The outranking approach and the foundations of ELECTRE methods', Theory and Decision, Vol. 31, No. 1, pp.49-73. 
Roy, B. (1996) Multicriteria Methodology for Decision Aiding, Kluwer Academic, Dordrecht.

Sadok, W., Angevin, F., Bergez, J., Bockstaller, C., Colomb, B., Guichard, L., Reau, R. and Dore, T. (2008) 'Ex ante assessment of the sustainability of alternative cropping systems: implications for using multi-criteria decision-aid methods. A review', Agronomy for Sustainable Development, Vol. 28, No. 1, pp.163-174.

Sadok, W., Angevin, F., Bergez, J., Bockstaller, C., Colomb, B., Guichard, L., Reau, R. and Messean, A. and Dore, T. (2009) 'MASC, a qualitative multi-attribute decision model for ex ante assessment of the sustainability of cropping systems', Agronomy for Sustainable Development, Vol. 29, No. 3, pp.447-461.

Słowiński, R., Greco, S. and Matarazzo, B. (2002) 'Axiomatization of utility, outranking and decision-rule preference models for multiple-criteria classification problems under partial inconsistency with the dominance principle', Control and Cybernetics, Vol. 4, No. 31, pp.1005-1035.

Sobrie, O., Mousseau, V. and Pirlot, M. (2012) 'Learning the parameters of a multiple criteria sorting method from large sets of assignment examples', in DA2PL 2012 Workshop from Multiple Criteria Decision Aid to Preference Learning, pp.21-31, Mons, Belgique [online] http://www.lgi.ecp.fr/DA2PL (access 30 May 2014).

Sobrie, O., Mousseau, V. and Pirlot, M. (2013) 'Learning a majority rule model from large sets of assignment examples', in Perny, P., Pirlot, M. and Tsoukias, A. (Eds.): ADT2013 Algorithmic Decision Theory, LNAI, Vol. 8176, pp.336-350, Springer, Heidelberg.

Stefanowski, J. (1998a) 'On rough set based approaches to induction of decision rules', in Skowron, A. and Polkowski, L. (Eds.): Rough Sets in Data Mining and Knowledge Discovery, Vol. 1, pp.500-529, Physica-Verlag.

Stefanowski, J. (1998b) 'Rough set based rule induction techniques for classification problems', in Proc. 6th European Congress on Intelligent Techniques and Soft Computing, Vol. 1, pp.109-113.

Stefanowski, J. (2002) 'Bagging and induction of decision rules', in Verlag, P. (Ed.): Int. Symposium on Intelligent Systems, pp.121-130.

Tardieu, F. (2003) 'Virtual plants: modelling as a tool for the genomics of tolerance to water deficit', Trends in Plant Science, Vol. 8, No. 1, pp.9-14.

Zak, J. (2009) 'Multiple criteria evaluation and optimization of transportation systems', Journal of Advanced Transportation, Vol. 43, No. 2, pp.91-94. 


\section{Appendix}

\section{Example of obtained decision rules}

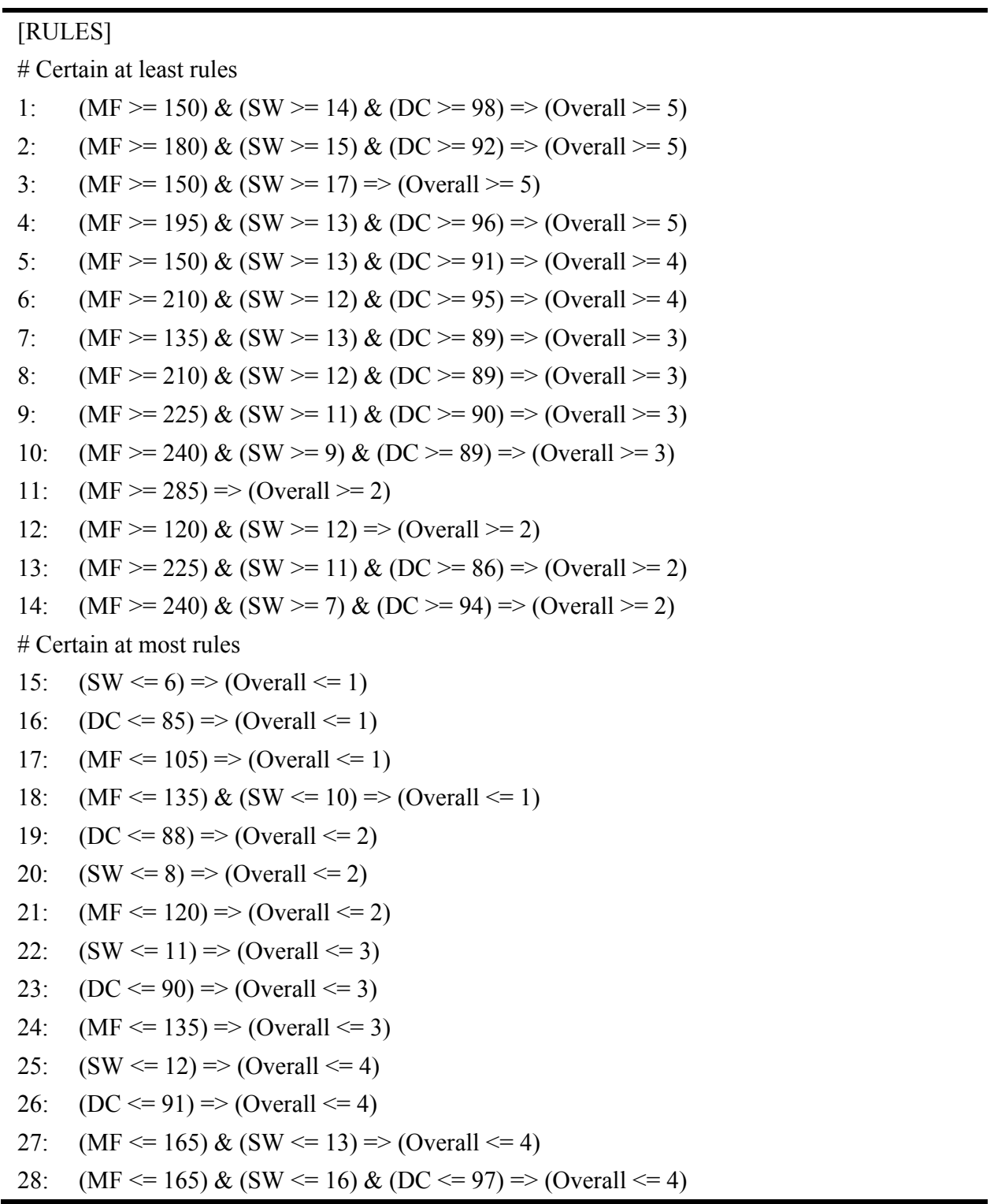

